\title{
Article
}

\section{Metabolomic Characterizations of Fullerene Nanomaterials Potentiate Muscle of Clownfish (Amphiprion Ocellaris)}

\author{
Xiaomei Huang ${ }^{1}, 2 \uparrow$, Meiying Zhao ${ }^{1} \uparrow$, Shan Lin ${ }^{3,4} \uparrow^{*}$, Min $\mathrm{Xu}^{1}$, Linchun $\mathrm{Li}^{1 *}$ \\ 1 Department of Marine Biology, Xiamen Ocean Vocational College, Xiamen, Fujian, 361100, P.R. China, \\ 2 Shanghai Institutes of Nutrition and Health, Chinese Academy of Sciences, Shanghai, 200031, P.R. China, \\ 3 Department of Orthopaedics, the First Affiliated Hospital of Xiamen University, Xiamen, Fujian, 361003, \\ P.R. China, \\ 4 School of Medicine, Xiamen University, Xiamen, Fujian, 361102, P.R. China, \\ * Correspondence: 2018642046@xmu.edu.cn; lilinchun@xmoc.edu.cn \\ $\dagger$ These authors contribute equally to this work.
}

\begin{abstract}
Fullerene nanomaterials exposure often causes a variety of diseases. Many studies have pointed out that fullerene nanomaterials can be studied in fish. However, there are few studies on health risk assessment of clownfish with lower doses of fullerene nanoparticles or different exposure durations. In this study, we set $1.5 \%$ and $3.0 \%$ for low- and high-dose fullerene nanomaterials exposure concentrations, respectively. Meanwhile, we performed a time-series analysis to investigate that the activation of lipid and amino acids metabolism after fullerene nanomaterials exposure in clownfish. 1368 metabolites were detected from clownfish by using liquid chromatography-mass spectrometry (LC-MS) analyses. Our results suggest that exposure to lower fullerenes nanoparticles may have a certain promoting effect on clownfish overall length, body length and weight. At the same time, the activation of potential metabolic pathways enriched by different metabolites in KEGG pathway may also indicate the positive promoting effect of fullerene nanoparticles after exposure. The present work indicates that it is particularly important to find the concentration window for fullerene nanomaterials to improve government safety guidelines, especially when these are applied to assess the health risk of human.
\end{abstract}

Keywords: fullerene; nanomaterials; clownfish; metabolomic; muscle

\section{Introduction}

Manufactured nanomaterials (MNMs) are defined as critical dimensions less than $100 \mathrm{~nm}$ at least one geometric surface, and a material with high uniformity. In particular, a product that has been artificially produced for the purpose of application[1]. Because MNMs have many excellent and peculiar physical or chemical properties, it is widely used in medicine, industry, construction, food and other fields. Fullerene Nanomaterials are one of the most widely used MNMs. Fullerenes are general term for a substance with a closed cage structure. It is an all-carbon molecule consisting of 12 five-membered rings and several six-membered rings[2]. As a new type of carbon material, fullerene have shown excellent properties in superconductivity, solar cells, nanometer electronic devices, catalysis and biology and have broad application prospects[3]. Over the past 20 years, the research on fullerenes has made great progress in both basic research and application fields.

The impact of fullerene nanomaterials on animals is relatively large. Some studies indicate that water-soluble fullerene is a good choice for detoxification and free radical absorption. Ishii et al. evaluated the histology of rat tibial muscles by using different type of water-soluble fullerene. The results showed that fullerene could promote the regeneration of skeletal muscle[4]. Metabolites are small molecule of less than $1000 \mathrm{Da}$ and can be involved in cellular metabolic reactions. The number of metabolites in cell, 
tissues, and humans ranges from hundreds to tens of thousands[5-7]. Because these metabolites have different chemical properties, it is difficult to detect all the metabolites or target the metabolites by conventional analytical methods. Metabolomics can solve this problem very well. After genomics, transcriptomics and proteomics, metabolomics is an important part of systems biology[8]. In the dynamic process of metabolism, metabolomics can systematically study the changing rules of metabolites and reveal the metabolic nature of life activities of the organism[9]. Metabolomics is widely used in fish research and its future potential. These results suggest that at least some energy is needed during swimming, in other words, several processes are involved in ATP production in turbot spermatozoa. Viant et al. assessed the metabolic conditions of juvenile steelhead trout (Oncorhynchus mykiss) at 15 and $20^{\circ} \mathrm{C}$ for a 10 -week exposure[10]. White muscle phosphocreatine, ATP, ADP and AMP, hepatic glycogen, and global metabolite profiling of muscle and liver were measured using $1 \mathrm{H}$ nuclear magnetic resonance (NMR) spectroscopy. These results showed that there was a positive correlation between increased stress and decreased metabolic condition. In addition, the data of growth showed $20^{\circ} \mathrm{C}$ is the upper limit of the steelhead trout's preferred range. Of course, there are other studies that apply metabolomics to fish[11-13]. In general, metabolomic has proved to be very useful for addressing a wide variety of hypotheses relating to fish physiology and development. In this study, we investigated the effects of different concentrations of fullerenes on the growth of clownfish and metabolomic analysis of its fish composition.

\section{Results}

\subsection{Effects of Fullerene Nanomaterials on Overall Length, Body Length and Weight Index of} Clownfish

As shown in Fig 1A, sampling results after 28 days of exposure showed that exposure to low-dose fullerene nanomaterials resulted in significant increases in overall length, body length and weight. At the same time, high dose exposure also promoted the increase of overall length, body length and weight, and significantly affected the change of body weight. Samples after 56 days of exposure showed that different concentrations of fullerene exposure could promote significant increases in overall length, body length and weight indexes. As shown in Fig 1B, the results of physiological indexes of clownfish under the same treatment concentration at different time points showed that the overall length, body length and weight of clownfish in the control group showed no significant changes at two time points, while the weight of clownfish showed no significant changes before and after the comparison. However, the overall length and body length of the low-dose group changed significantly at 2 time points after exposure, and the influence of high-dose exposure on body length was obvious. In general, the effects of low dose fullerene nanoparticles on overall length, body length and weight of clownfish were more obvious than those of high dose, and all of them showed an upward trend. The experimental results in Fig. 1B also suggested that the influence of fullerene nanoparticles on overall length and body length was more obvious than that of weight. 

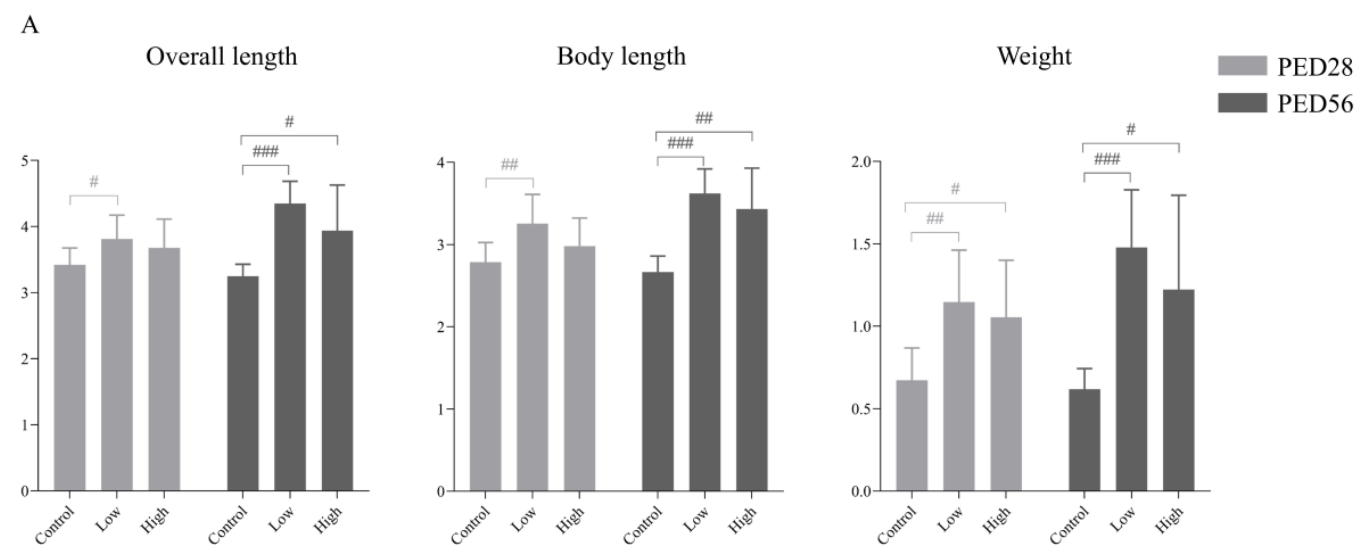

B

Overall length
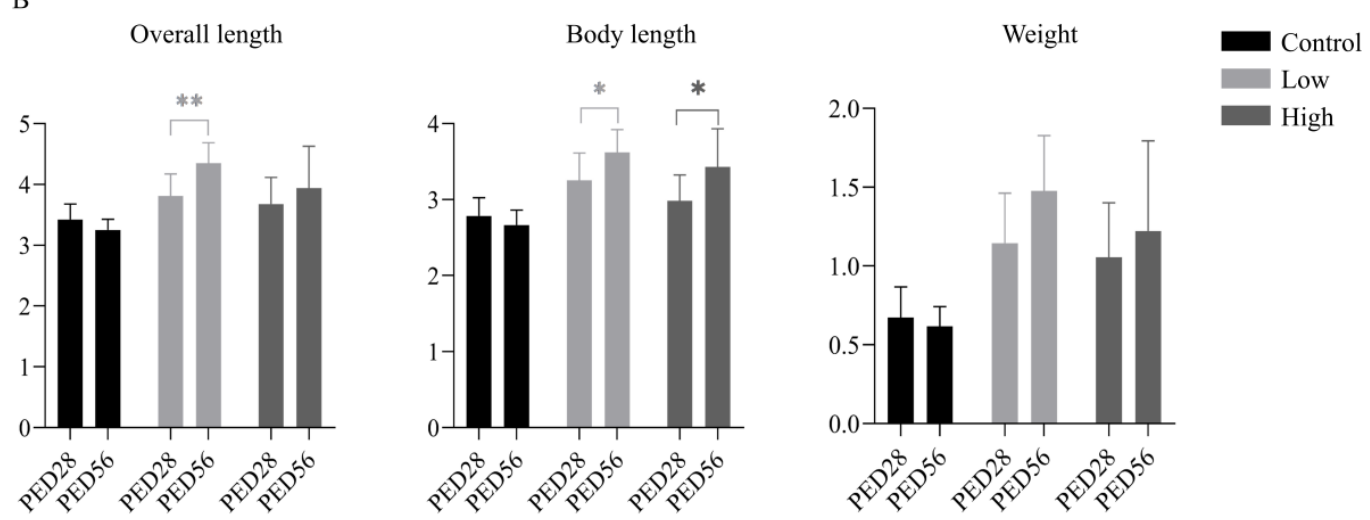

Figure 1. The results of overall length, body length and weight (A: light grey: after 28 days of exposure; dark grey: after 56 days of exposure; B: black: control; light grey: low-dose group; dark grey: high-dose group). ${ }^{*} \mathrm{P}<0.05,{ }^{* *} \mathrm{P}<0.01$, ${ }^{* * *} \mathrm{P}<$ 0.001 .

\subsection{Principal Component Analysis of Muscle Metabolomics in Clownfish}

Principal component analysis (PCA) can deal with multicollinearity problem well. We used PCA model to study the metabolomics of clownfish muscles under different concentrations and durations of fullerene exposure. The results of PCA score plot showed that the first and second principal components accounted for $20.9 \%$ and $13.1 \%$ of the variation, respectively (Figure 2). The sample of the PED 28 and PED 56 were clearly distinguished in the first principal component, it indicated that the correlation between PED 28 and PED 56 samples was weak.

In order to explore whether exposure to different concentrations of fullerene nanoparticles changes the muscle metabolomics level of clownfish independently of time, unsupervised PCA analysis was performed on samples at two different exposure time points (Figure 2B, 2C). On PED 28, the first two principal components explained $23.4 \%$ and $10.5 \%$ of the variation, respectively. Different exposure groups were distinguished from the control group on the first principal component, and the correlation between the high-dose group and the control group, the low-dose group and 
the control group was week. On PED 56, the results were similar on PED 28, the first two principal components accounted for $26.6 \%$ of the variation, with the first component being more differentiated than the second principal component. At the same time, the distance between the high-dose exposure group and the other two groups was far, and the correlation was weak. In conclusion, different concentrations of fullerene nanomaterials have different effects on muscle metabolic profile of clownfish, and the effect of high dose is more obvious. At the same time, the metabolic profile of clownfish muscle will change significantly under different exposure duration.

A

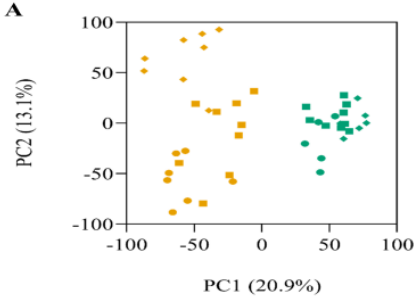

C

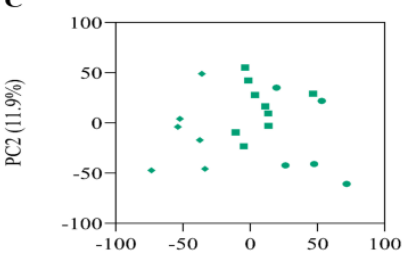

B

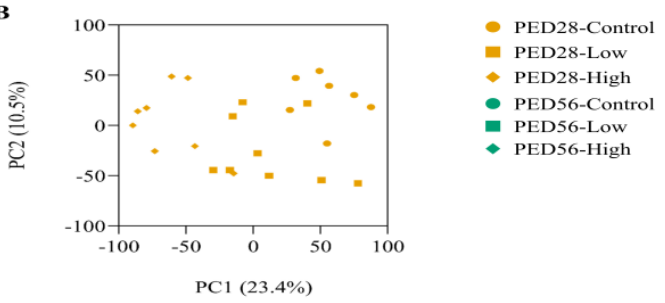

Figure 2. The results of principal component analysis (PCA). A: Dynamic change of metabolites expression over time; B: result of PED28; C: result of PED56.

\subsection{Multivariate analysis of the effects of Fullerene Nanomaterials exposure in Clownfish metabolomics fingerprint analysis}

PLS-DA model was used to analyze and visualize the "exposed group-control group" supervision data of clownfish muscle tissue at different time points. Results of 200 replacement tests proved that the model was robust and reliable. As shown in Figure 3A-D, the PLS-DA score of clownfish muscle was changed by exposure at different concentrations (Figure 3), and the distance between points represented the similarity between different samples. Although the results in different groups suggested differences between individuals, the exposed group and the control group were clearly differentiated, with obvious clustering on the first principal component (PC1). Compared with the control group, the PLS-DA model had the best modeling and predictive power for all exposure groups (Figure 3A: $\mathrm{R} 2 \mathrm{X}=0.409, \mathrm{R} 2 \mathrm{Y}=0.996, \mathrm{Q} 2=0.818$; Figure 3B: $\mathrm{R} 2 \mathrm{X}=0.422, \mathrm{R} 2 \mathrm{Y}=0.996, \mathrm{Q} 2=0.945$; Figure 3C: $\mathrm{R} 2 \mathrm{X}=0.251, \mathrm{R} 2 \mathrm{Y}=0.966, \mathrm{Q} 2=0.526$; Figure 3C: $R 2 X=0.251, R 2 Y=0.966, Q 2=0.526$; Figure 3D.. $R 2 X=0.334, R 2 Y=0.993$, $\mathrm{Q} 2=0.796$ ). The obvious clustering between the control and exposed samples indicated that exposure to fullerene nanomaterials induced changes in muscle metabolism of clownfish. In this study, VIP represents the importance of variables that significantly change the exposure response of fullerene nanomaterials, and we screened important metabolites by setting VIP greater than 1.0. 
A

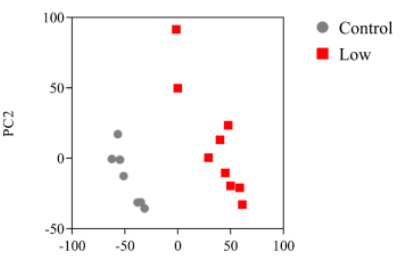

$\mathrm{PCl}$

C

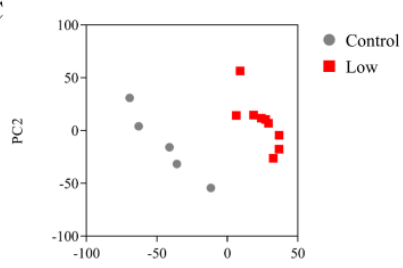

PC1
B

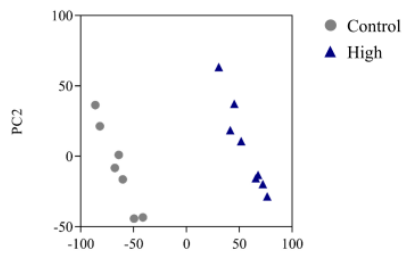

$\mathrm{PCl}$

D

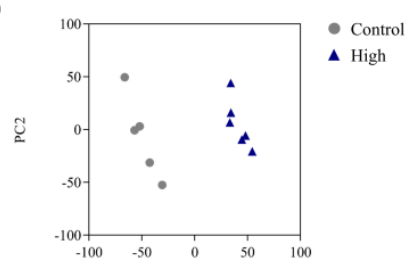

PC1

Figure 3. The results of PLS-DA models (A: PED28 L vs. C; B: PED28 H vs. C; C: PED56 L vs. C; D: PED56 H vs. C).

A

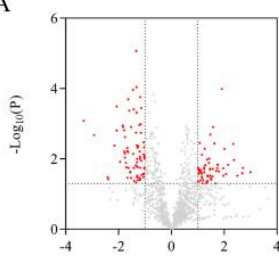

$\log _{2}(\mathrm{FC})$

D

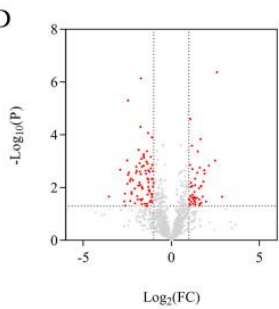

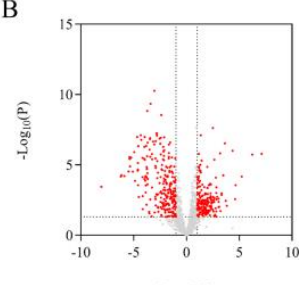

$\log _{2}(\mathrm{FC})$

E

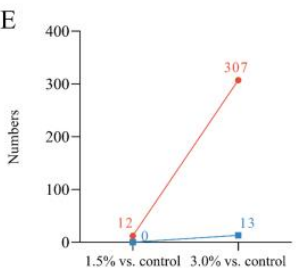

C

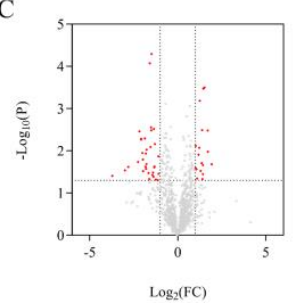

Figure 4. The volcano plots of metabolites in different groups. (A: PED28 L vs. C; B: PED28 H vs. C; C: PED56 L vs. C; D: PED56 H vs. C; E: numbers of metabolites corrected by P value).

\subsection{Biopathway enrichment analysis of differential metabolites}

In order to further screen the metabolites with significant changes after exposure to fullerene nanomaterials, $\mathrm{P}<0.05$ and $\mathrm{Fc}>2.0$ were combined to screen the metabolites with significant changes at different time points and at different exposure concentrations (Figure 5). We found that 133, 499; 52, 130 metabolites were screened in L vs. C and H vs. C comparison groups of PED 28 and PED 56, respectively. At the same time, we combined the previous VIP threshold screening results and corrected the P values, and finally screened 12,$307 ; 0,13$ significantly altered differential metabolites from the L vs. $\mathrm{C}$ and $\mathrm{H}$ vs. $\mathrm{C}$ groups of PED 28 and PED 56 respectively. Through statistical analysis of the significant changes in the number of differential metabolites, it was found that with the increase of the exposure concentration of fullerene nanomaterials, the number of significant changes in the number of metabolites increased. These significantly changing differential metabolites were combined and screened based on the HMDB database, and 39 kinds of endogenous differential metabolites with significant changes were finally 
identified, as shown in Figure 5A. Consistent with the PLS-DA score, Fig. 5A shows a relatively obvious clustering between the control and exposed samples.

In addition, the significantly changed differential metabolites screened were imported into the Pathway Analysis module of Metaboanalyst 5.0, and the metabolic Pathway enrichment Analysis of the significantly changed differential metabolites was conducted (Figure 5B). The results showed that exposure to fullerene nanoparticles could induce changes in biotin metabolism, glycerol phosphate shuttling, triglyceride biosynthesis and cardiolipin biosynthesis.

A

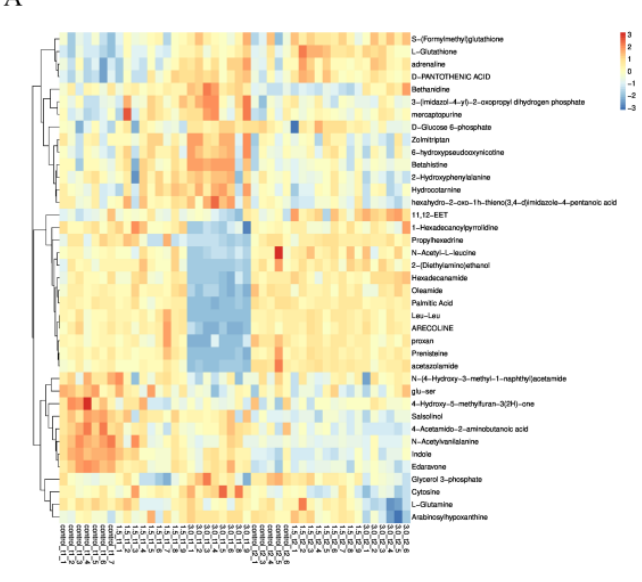

B

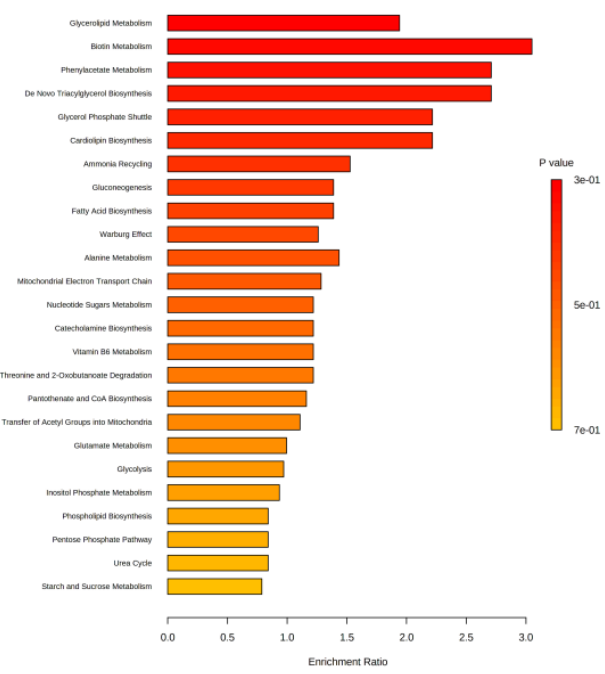

Figure 5. Heat map of differential metabolites and result of metabolites enrichment.

\section{Discussion}

Like mice and other mammals, clownfish have well-developed defense system[14]. Weight, overall length and body length are important index of clownfish growth state. Previous studies investigated that different concentrations fullerene nanomaterials can induce reproductive toxicity in freshwater fish[15], nanoparticles can enter cells to produce cytotoxicity [16-18], and hydroxylated fullerenes in rat liver lead to cell death and decreased ATP levels[19]. However, there is a close relationship between pollutant exposure concentration and health. At present, most studies have focused on the harmful effects of nanomaterials on health[14, 20], it was found in this study that exposure to different concentrations can activate and promote the overall length, body length and weight of clownfish. Fullerene nanoparticles are widely used in industrial catalysts, lubricants and superconductors[21], and in drug delivery[22]. Therefore, it is particularly necessary to study the biological effects of low dose exposure, which can provide credible evidence for the study of toxicity mechanism and improve government safety guidelines.

Based on unsupervised PCA model analysis results, we found that different sampling points and different concentrations of fullerene nanoparticles could cause changes in the metabolic profile of clownfish. At the same time, according to KEGG metabolic pathway enrichment analysis of co-expressed metabolites in different tissues. It was found that different fullerene nanoparticles exposure would affect biological processes including arachidonic acid metabolism, fatty acid biosynthesis, pyrimidine metabolism and amino acid metabolism, et al. These data indicate that different metabolic processes exist in different clownfish after fullerene nanoparticles exposure and amino acid metabolism and lipid metabolism may be important molecular events of fullerene nanoparticles exposure under different environmental pollution conditions. Furthermore, the phosphopantetheinyl portion of $\mathrm{CoA}$ is a crucial component of acyl carrier protein, 
essential for the biosynthesis of fatty acid and lipid metabolism[23]. In our study, some lipids and lipid-like molecules were identified as core elements, these affected lipid metabolism mainly contain the general functions of energy storage, cell membrane composition and signal transmission and molecular recognition processes[24-26]. Fullerene nanoparticles stimulated the release of arachidonic acid from clownfish cells and arachidonic acid (AA) from membrane phospholipids via phospholipase A2S, which may be related to the pathophysiological events[27]. At the same time, fullerene nanoparticles can also cause changes in the amino acid metabolism of clownfish, and these metabolites are usually closely related to TCA cycle and energy metabolism. Our analysis of clownfish metabolites helps to screen out metabolites sensitive to the exposure of fullerene nanoparticles, which can be used to characterize the exposure level of fullerene nanoparticles existing in industry or other aspects, and is of great significance for health risk assessment.

\section{Materials and Methods}

\subsection{Preparation of Feed}

The feed used in this experiment was purchased from a well-known brand in China. Fullerene microcapsule powder is provided by Xiamen Funano New Material Technology Co.,Ltd. When the feed containing fullerene microcapsules powder was prepared, the powder was dissolved in sterile water to make 3 groups of solutions containing $0 \%, 1.5 \%$ and $3.0 \%$ of fullerene, which were evenly sprayed on the surface of the formula feed by spray technology. After natural drying, the powder was stored in a refrigerator at $-20^{\circ} \mathrm{C}$ for later use.

\subsection{Experimental Design}

The experiment was carried out in Xiamen Ocean Vocational and Technical College. Clownfish for the experiment were purchased from Xiamen Fish Home Ornamental Fish Farm. Before the experiment, cement ponds and net cages were disinfected with bleach powder. The young fish fry was transported to the laboratory and temporarily raised for 4 weeks. In the last 1 week, they were domesticated with basic feed. After temporary breeding, 540 healthy clownfish with a body weight of $(0.78 \pm 0.08)$ g were randomly assigned to 9 aquariums, and the experiment was divided into 3 groups, 3 in each group and 30 in each parallel group. Feed twice a day (09:00 and 16:00) with full food for about 30 minutes, and then remove residual bait and feces. Water quality indexes were measured weekly. During culture, the water temperature was $(26 \pm 3.5){ }^{\circ} \mathrm{C}, \mathrm{pH} 8.5 \pm 0.5$, dissolved oxygen $4.0 \sim 5.0 \mathrm{mg} / \mathrm{L}$ and ammonia nitrogen $0 \sim 0.03 \mathrm{mg} / \mathrm{L}$. The culture experiment lasted for 60 days.

\subsection{Sample Collection and Pretreatment}

Clownfish were starved for 24h on the 28th (PED28) and 56th (PED56) days after feeding fullerene-containing diet, respectively. Nine fish were randomly selected from each group. After being anesthetized on the ice tray, the body length and body weight of the individuals were measured and stored at $-80^{\circ} \mathrm{C}$ for metabolomic analysis of fish composition.

The method of samples pretreatment in this study was adjusted according to Elizabeth J Want28 and Warwick B Dunn 's29 samples handling method. Muscle tissue of fish were weighed accurately and put into Suizhen crushing tube, then added $1 \mathrm{~mL}$ pre-cooled methanol: water (v: $v=1: 1$ ) to each tube. It was crushed for 30s (Fastpre-24, USA), centrifuged for $15 \mathrm{~min}\left(15800 \mathrm{~g}, 15 \mathrm{~min}, 4^{\circ} \mathrm{C}\right)$, then absorbed supernatant to the new centrifugal tube. The supernatants were dried under vacuum (Thermo, RVT4104) before derivatization for LC-MS. Before the test, all samples were re-dissolved in $100 \mu \mathrm{L}$ of precooled methanol: water (v: v=1:1) and centrifuged at $15800 \mathrm{~g}$ at $4^{\circ} \mathrm{C}$ for $15 \mathrm{~min}$ and absorbed for use on the machine. 
The LC-MS test was carried out on a Waters UPLC-class system with a sample manager and a binary solvent delivery manager, coupling with a Thermo Q-Exactive Mass Spectrometer equipped with an electrospray interface (Thermo Scientific, USA). LC was performed using HSS T3 $(1.8 \mu \mathrm{m}, 2.1 \mathrm{~mm}$ i.d. $\times 100 \mathrm{~mm})$ column (Waters Technologies). $0.1 \%$ formic acid was added to Solvents $\mathrm{H} 2 \mathrm{O}$ (A) and Methanol (B), simultaneously. Column was heated to $50^{\circ} \mathrm{C}$ and the auto-sampler was regulated to $8^{\circ} \mathrm{C} .10 \mu \mathrm{L}$ injection were separated under gradient conditions at a flow rate of $300 \mu \mathrm{L} / \mathrm{min}$. The gradient was as follows: serum elution gradient was that $0 \%$ (B), keep 1min, over 1-16.0 $\min , 0 \%-100 \%$ (B), keep $100 \%$ (B) to $20 \mathrm{~min}, 100 \%-0 \%$ (B) over $20.1-22.0 \mathrm{~min}$. The mass spectrometer was operated in full-scan mode with a range of 100 1000 m/z. Spray voltage was set at $3500 \mathrm{~V}$ and capillary temperature was $320^{\circ} \mathrm{C}$. To carry out MS/MS mode to identify differentiated metabolites, argon was used as a collision gas and the collision energy was adjusted from $15 \mathrm{eV}$ to $40 \mathrm{eV}$ for each metabolome.

\subsection{Metabolomics Data Processing}

In order to eliminate the problem that the signal strength of the LC-MS drift with time during the detection process, Compound Discoverer 3.1(CD 3.1) was used to align raw LC-MS data [28]. The obtained data matrix provided information about mass-to-charge ratio $(\mathrm{m} / \mathrm{z})$, sample information, peak intensity and retention time, etc. After data information extraction was completed by using CD 3.1 software, and QC-RLSC [29]calibration was used to carry out with the installation 'statTarget' package of $R[30,31]$. After correction of QC, those metabolites with RSD greater than 0.2 were deleted and normalized by summation. The normalized data sets were subjected to multivariate statistical analysis using SIMCA software (Version 14.1, Umetrics, Umeå, Sweden) and/or MetaboAnalyst 5.0[32,33]including the principle component analysis (PCA) and the partial least-squares discriminant analysis (PLS-DA), which are developed specifically for the analysis of omics datasets[34]. Specifically, the outliers were identified using the principle component analysis (PCA) with mean-center scaling. The PLS-DA with pareto scaling (par) scaling was then carried out to extract the differential metabolites between $\mathrm{L}$ vs. $\mathrm{C}$ and $\mathrm{H}$ vs. $\mathrm{C}$ groups. In this study, we identified VIP $>1.0$, Adjusted-p $<0.05$, Fold-change $(\mathrm{FC})>2$ or $<0.5$ as differential metabolites.

\subsection{Statistical Analysis}

The statistical analysis was performed using MetaboAnalyst 5.0. All the data was analyzed using Non-parametric Mann-Whitney U tests. In all cases, adjusted-p $<0.05$ was considered as statistically significant. Graphics were done using GraphPad Prism (Version 8, La Jolla, CA, USA).

\section{Conclusions}

Many studies have linked exposure to fullerene nanoparticles to metabolic disorders, and more studies have focused on the relationship between exposure to fullerene nanoparticles and adverse health outcomes. However, it is still unclear whether low exposure concentration has a positive effect on metabolism. In this study, we simulated the actual environment of clownfish by setting different exposure duration and exposure concentration. Through the analysis of the metabolic results of clownfish, it was found that low dose of fullerene nanoparticles exposure may enhance the metabolic level of clownfish. A variety of different metabolites were identified, which were mainly involved in lipid and amino acid metabolism. In general, this study can provide reliable data support for the toxicity study of fullerene nanoparticles.

Author Contributions: Conceptualization, X.H. and L.L.; methodology, M.Z.; software, X.H.; validation, X.H., M.Z. and S.L.; formal analysis, L.L.; investigation, M.Z.; resources, X.H.; data curation, S.L.; writing-original draft preparation, S.L.; writing-review and editing, X.H.; visualization, S.L.; supervision, L.L.; project administration, M.X.; funding acquisition, M.X. All authors have read and agreed to the published version of the manuscript. 
Acknowledgments: This research was funded by Marine Organism Application Technology Collaborative Innovation Center Project (NO. XTZX-HYSW-1801), Fujian Educational and Scientific Research Program for Young and Middle-aged Teachers (NO. JAT191308, NO. JAT191320), Xiamen Ocean Vocational College: Research Projects for High-level Talents (NO. 140008).

Conflicts of Interest: The authors declare no conflict of interest.

\section{References}

1. Colvin VL: The potential environmental impact of engineered nanomaterials. Nature biotechnology 2003, 21(10):1166-1170.

2. Kroto HW, Heath JR, O’Brien SC, Curl RF, Smalley RE: C 60: buckminsterfullerene. nature 1985, 318(6042):162-163.

3. Oberdörster E: Manufactured nanomaterials (fullerenes, C60) induce oxidative stress in the brain of juvenile largemouth bass. Environmental health perspectives 2004, 112(10):1058-1062.

4. Ishii A, Yoshida M, Ohkoshi N, Ueno H, Kokubo K, Tamaoka A: GP 204: The effect of water-soluble fullerene in muscle regeneration process. Neuromuscular Disorders 2014, 24(9):878.

5. Famili I, Förster J, Nielsen J, Palsson BO: Saccharomyces cerevisiae phenotypes can be predicted by using constraintbased analysis of a genome-scale reconstructed metabolic network. Proceedings of the National Academy of Sciences 2003, 100(23):13134-13139.

6. Wishart DS, Tzur D, Knox C, Eisner R, Guo AC, Young N, Cheng D, Jewell K, Arndt D, Sawhney S: HMDB: the human metabolome database. Nucleic acids research 2007, 35(suppl_1):D521-D526.

7. Goodacre R: Making sense of the metabolome using evolutionary computation: seeing the wood with the trees. Journal of experimental botany 2005, 56(410):245-254.

8. Nicholson JK, Timbrell JA, Sadler PJ: Proton NMR spectra of urine as indicators of renal damage. Mercury-induced nephrotoxicity in rats. Molecular Pharmacology 1985, 27(6):644-651.

9. Dreanno C, Seguin F, Cosson J, Suquet M, Billard R: 1H - NMR and 31P - NMR analysis of energy metabolism of quiescent and motile turbot (Psetta maxima) spermatozoa. Journal of Experimental Zoology 2000, 286(5):513-522.

10. Allen PJ, Wise D, Greenway T, Khoo L, Griffin MJ, Jablonsky M: Using 1-D 1 H and 2-D 1 H J-resolved NMR metabolomics to understand the effects of anemia in channel catfish (Ictalurus punctatus). Metabolomics 2015, 11(5):1131-1143.

11. Samuelsson LM, Larsson DJ: Contributions from metabolomics to fish research. Molecular BioSystems 2008, 4(10):974-979.

12. Southam AD, Easton JM, Stentiford GD, Ludwig C, Arvanitis TN, Viant MR: Metabolic changes in flatfish hepatic tumours revealed by NMR-based metabolomics and metabolic correlation networks. Journal of proteome research 2008, 7(12):52775285.

13. Du H, Fu J, Wang S, Liu H, Zeng Y, Yang J, Xiong S: 1 H-NMR metabolomics analysis of nutritional components from two kinds of freshwater fish brain extracts. Rsc Advances 2018, 8(35):19470-19478.

14. Zhu X, Zhu L, Lang Y, Chen Y: Oxidative stress and growth inhibition in the freshwater fish Carassius auratus induced by chronic exposure to sublethal fullerene aggregates. Environmental Toxicology and Chemistry: An International Journal 2008, 27(9):1979-1985.

15. Sumi N, Chitra K: Fullerene C60 nanomaterial induced oxidative imbalance in gonads of the freshwater fish, Anabas testudineus (Bloch, 1792). Aquatic Toxicology 2019, 210:196-206.

16. Foley S, Crowley C, Smaihi M, Bonfils C, Erlanger BF, Seta P, Larroque C: Cellular localisation of a water-soluble fullerene derivative. Biochemical and biophysical research communications 2002, 294(1):116-119.

17. Sayes CM, Gobin AM, Ausman KD, Mendez J, West JL, Colvin VL: Nano-C60 cytotoxicity is due to lipid peroxidation. Biomaterials 2005, 26(36):7587-7595.

18. Porter AE, Muller K, Skepper J, Midgley P, Welland M: Uptake of C60 by human monocyte macrophages, its localization and implications for toxicity: studied by high resolution electron microscopy and electron tomography. Acta biomaterialia 2006, 2(4):409-419. 
19. Nakagawa Y, Suzuki T, Ishii H, Nakae D, Ogata A: Cytotoxic effects of hydroxylated fullerenes on isolated rat hepatocytes via mitochondrial dysfunction. Archives of toxicology 2011, 85(11):1429-1440.

20. Shinohara N, Matsumoto T, Gamo M, Miyauchi A, Endo S, Yonezawa Y, Nakanishi J: Is lipid peroxidation induced by the aqueous suspension of fullerene $\mathbf{C} 60$ nanoparticles in the brains of Cyprinus carpio? Environmental science $\mathcal{E}$ technology 2009, 43(3):948-953.

21. Yudoh K, Karasawa R, Masuko K, Kato T: Water-soluble fullerene (C60) inhibits the osteoclast differentiation and bone destruction in arthritis. International journal of nanomedicine 2009, 4:233. Arbogast JW, Darmanyan AP, Foote CS, Diederich F, Whetten R, Rubin Y, Alvarez MM, Anz SJ: Photophysical properties of sixty atom carbon molecule (C60). The Journal of Physical Chemistry 1991, 95(1):11-12. Leonardi R, Zhang Y-M, Rock CO, Jackowski S: Coenzyme A: Back in action. Progress in Lipid Research 2005, 44(2):125-153. van Meer G, Voelker DR, Feigenson GW: Membrane lipids: where they are and how they behave. Den Besten G, Van Eunen K, Groen AK, Venema K, Reijngoud DJ, Bakker BM: The role of short-chain fatty acids in the interplay between diet, gut microbiota, and host energy metabolism. Journal of Lipid Research, 54(9):2325-2340. Huang Y, Sun F, Tan H, Deng Y, Sun Z, Chen H, Li J, Chen D: DEHP and DINP Induce Tissue-and Gender-Specific Disturbances in Fatty Acid and Lipidomic Profiles in Neonatal Mice: A Comparative Study. Environmental Science $\mathcal{E}$ Technology 2019, 53(21):12812-12822.

27. Pozzi R, De Berardis B, Paoletti L, Guastadisegni C: Inflammatory mediators induced by coarse (PM2.5-10) and fine (PM2.5) urban air particles in RAW 264.7 cells. Toxicology 2003, 183(1):243-254.

28. Swortwood MJ, Carlier J, Ellefsen KN, Wohlfarth A, Diao X, Concheiro-Guisan M, Kronstrand R, Huestis MA: In vitro, in vivo and in silico metabolic profiling of $\alpha$-pyrrolidinopentiothiophenone, a novel thiophene stimulant. Bioanalysis 2016 , $8(1): 65-82$.

29. Dunn WB, Broadhurst D, Begley P, Zelena E, Francis-McIntyre S, Anderson N, Brown M, Knowles JD, Halsall A, Haselden JN: Procedures for large-scale metabolic profiling of serum and plasma using gas chromatography and liquid chromatography coupled to mass spectrometry. Nature protocols 2011, 6(7):1060.

30. Luan H, Ji F, Chen Y, Cai Z: statTarget: A streamlined tool for signal drift correction and interpretations of quantitative mass spectrometry-based omics data. Analytica chimica acta 2018, 1036:66-72.

31. Luan H, Ji F, Chen Y, Cai Z: Quality control-based signal drift correction and interpretations of metabolomics/proteomics data using random forest regression. bioRxiv 2018:253583. Chong J, Soufan O, Li C, Caraus I, Li S, Bourque G, Wishart DS, Xia J: MetaboAnalyst 4.0: towards more transparent and integrative metabolomics analysis. Nucleic Acids Research 2018, 46(W1):W486-W494.

33. Xia J, Wishart DS: Web-based inference of biological patterns, functions and pathways from metabolomic data using MetaboAnalyst. Nature Protocols, 6(6):743-760.

34. Madsen R, Lundstedt Tr, Trygg J: Chemometrics in metabolomics-A review in human disease diagnosis. 659(1-2):23-33. 